\title{
Nasopharyngeal metastasis from colorectal cancer: a case report
}

\author{
Ye-Huan Liu1" ${ }^{\#}$, Bei-Bei Lin ${ }^{\prime \prime}$, Shi-Xu Lv² \\ ${ }^{1}$ Department of General Medicine, The First Affiliated Hospital of Wenzhou Medical University, Wenzhou, China; ${ }^{2}$ Department of Thyroid \& \\ Breast Surgery, The First Affiliated Hospital of Wenzhou Medical University, Wenzhou, China \\ "These authors contributed equally to this work. \\ Correspondence to: Shi-Xu Lv. Department of Thyroid \& Breast Surgery, The First Affiliated Hospital of Wenzhou Medical University, South of Bai- \\ xiang Street, Ou-hai District, 325000, Wenzhou, China. Email: lvshixu@foxmail.com.
}

\begin{abstract}
Metastases from colorectal cancer can occur either through the lymphatic or by hematogenous spread. The most common metastatic sites are the lung and liver. Nasopharyngeal metastasis from colorectal cancer has never been previously reported in the literature on the internet databases we can found. In this paper, we present the case of a 79-year-old male suffering from adenocarcinoma of the rectum with distant metastases to the liver, lung, and nasopharynx. Over the previous 7 years, he had received treatment for rectal cancer including radical surgery (miles surgery), chemotherapy, hepatectomy, and pneumonectomy. After local nasopharyngeal radiotherapy, the patient's quality of life significantly declined and they eventually died of dyspnea caused by airway obstruction due to a nasopharyngeal mass after 7 months of palliative treatment involving pain relief from end-stage disease. Nasopharyngeal metastases from colorectal cancer are extremely rare in the clinic. To the best of our knowledge, this is the first case reporting this occurrence which not only extends the disease database but also warns doctors to pay more attention to these clinical scenarios. Strict monitoring of patients with colorectal cancer after primary treatment could lead to the early diagnosis of metastases and give patients better opportunities for treatment and improved prognosis.
\end{abstract}

Keywords: Nasopharyngeal metastasis; colorectal cancer

Submitted Aug 20, 2020. Accepted for publication Nov 25, 2020.

doi: 10.21037/apm-20-1656

View this article at: http://dx.doi.org/10.21037/apm-20-1656

\section{Introduction}

Metastases from colorectal cancer can occur either by the lymphatics or by hematogenous spread with the most common secondary sites being the lungs and liver. Atypical sites of metastases from colorectal cancer involve organs such as the spleen, thyroid gland, spermatic cord, and skeletal muscles (1-4). Even though metastases to these sites might occur as a feature of end-stage disease, metastasis to the nasopharynx from colorectal cancer has never previously been reported in the literature on the internet databases we can found. For the first time, we report a case of nasopharyngeal metastasis from colorectal cancer 7 years after radical surgery for rectal cancer (miles surgery). We also present a concise review of the histopathological and clinical aspects of this rare case and evaluate the diagnostic and therapeutic options based on our observations in the case.
We present the following article in accordance with the CARE reporting checklist (available at http://dx.doi. org/10.21037/apm-20-1656).

\section{Case presentation}

In October 2012, a 72-year-old man was admitted to our hospital with a chief complaint of passing bloody stools for 2 months without seeking medical attention. The patient denied any abdominal pain and tenesmus. He reported no existing or previous health problems. The patient recorded a $2 \mathrm{~kg}$ weight loss within the 6 months before presenting at our hospital. A colonoscopy revealed a budding tumor in the rectum that easily bled. Histopathological analysis of the biopsy showed chronic inflammation of the rectal mucosa with high-grade intraepithelial neoplasia. These features were consistent with a highly differentiated 


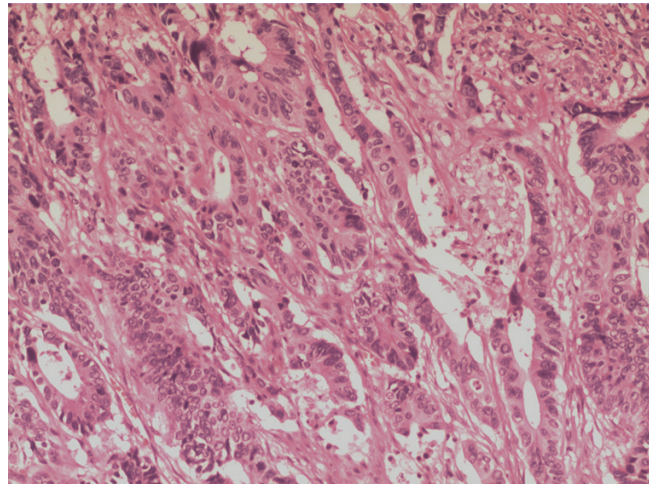

Figure 1 Tall malignant columnar cells lining large irregular glands (hematoxylin-eosin staining, magnification $\times 100$ ). Histopathological exam revealed an ulcerative type of moderately/ poorly differentiated adenocarcinoma. Immunohistochemical analysis displayed BcL-2 and KRAS negative staining with P53 positive staining.

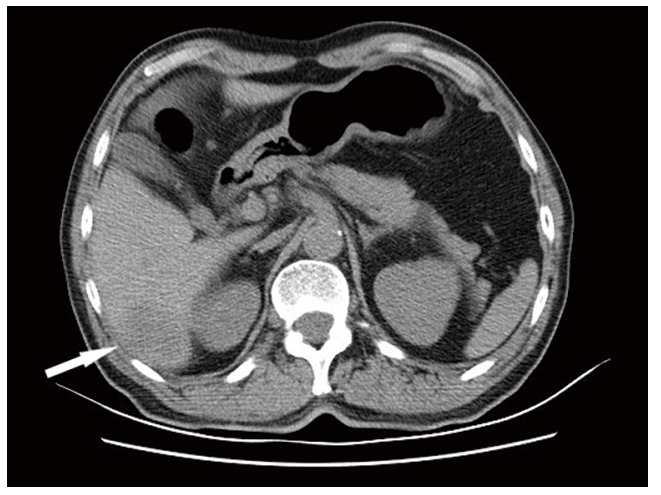

Figure 2 Abdominal CT scan demonstrating, in the horizontal view, a single low density lesion (arrow) in the right hepatic lobe (size $3.8 \times 2.8 \mathrm{~cm})$.

carcinoma. A computed tomography (CT) scan of the chest, abdomen, and pelvis, and brain magnetic resonance imaging (MRI) showed no abnormalities. He underwent radical surgery for rectal cancer (miles surgery) with a subsequent histopathological finding demonstrating an ulcerative type of moderately-poorly differentiated colorectal adenocarcinoma (size $3 \times 3 \mathrm{~cm}$ ). The tumor had infiltrated the layers of the intestinal wall and the adipose tissue outside the serous membrane. Four regional lymph nodes were resected and none showed metastases (pT4N0M0, Stage II). Immunohistochemical analysis showed the tissue was negative for Bcl-2 and KRAS, and positive for P53
(Figure 1). The status of MSI proteins and BRAF were not verified. There was no family history of colorectal cancer, malignancy, or familial predisposition for colorectal cancer. The operation was smooth and the postoperative recovery was good. Two months later, the patient received a chemotherapeutic regimen including oxaliplatin and capecitabine. However, oxaliplatin was discontinued due to a severe allergic reactions displayed at the first dose, and capecitabine was stopped following the appearance of a grade III hand-foot syndrome (HFS) after four cycles.

During the 7 years following surgical resection, the patient was followed up every 3 months with serological and radiological investigations for disease monitoring and detection of recurrence. Unfortunately, the patient underwent three operations during this period due to distant metastasis to the liver and lungs. In June 2014, he received a hepatectomy after a CT scan of the abdomen demonstrated a single low-density lesion in the right hepatic lobe (Figure 2). The patient subsequently underwent two endoscopic pulmonary wedge resections of isolated metastatic pulmonary lesions from the colorectal cancer that had been detected by a chest CT Scan in November 2016 and February 2017 (Figures 3,4). Blood tumor biomarker examinations were maintained within the normal range.

In August 2019, the 79-year-old man presented at his regular follow-up visit with headaches, diplopia, ptosis, and right-sided hearing loss which had been present for one week. During the physical examination, there was no enlargement of the cervical lymph nodes. Serum NSE and CYFRA21-1 were elevated with values of $17.9 \mathrm{ng} / \mathrm{mL}$ [0-15] and $17.7 \mathrm{ng} / \mathrm{mL}$ [0-13], respectively. Brain MRI displayed multiple lacunar infarctions within the brain. Also, a right bony mass was observed in the skull base and clivus involving the parapharyngeal space, cephalic longus muscle, and the cavernous sinus. Differential diagnosis was metastases or nasopharyngeal primary malignancies (Figure 5). Further examination by electronic nasopharyngoscopy revealed a large number of new cauliflower-like masses in the posterior roof wall of the nasopharynx and the right pharyngeal recess with its surface full of necrotic tissues. The texture of the tissue was brittle and it bled easily during biopsy (Figure 6). Histopathological examination demonstrated adenocarcinoma with extensive necrosis which was consistent with intestinal adenocarcinoma metastasis to the nasopharynx based on immunohistochemical results and clinical history. Immunohistochemical analysis showed the tissue was positive for CDX-2, focal TTF-1 and Villin, and negative for CK20 and Napsin A (Figure 7). Local nasopharyngeal 

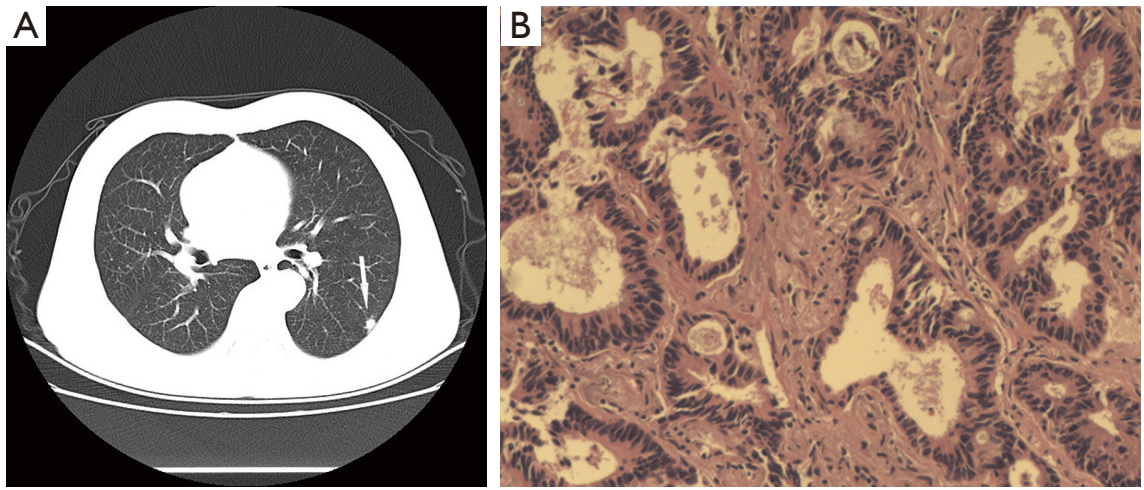

Figure 3 (A) Chest CT scan demonstrating, in the horizontal view, an isolated nodule of 9 mm diameter (arrow) in the lobe of the left lung. (B) Adenocarcinoma spread to the left lung parenchyma (hematoxylin-eosin staining, magnification $\times 100$ ). Histopathological exam revealed tubular adenocarcinoma with a small amount of necrosis. Immunohistochemical analysis displayed positive staining of CDX-2 (+++) Villin (++) and negative staining of CK20 (-), CK7 (-) and TTF-1 (-).
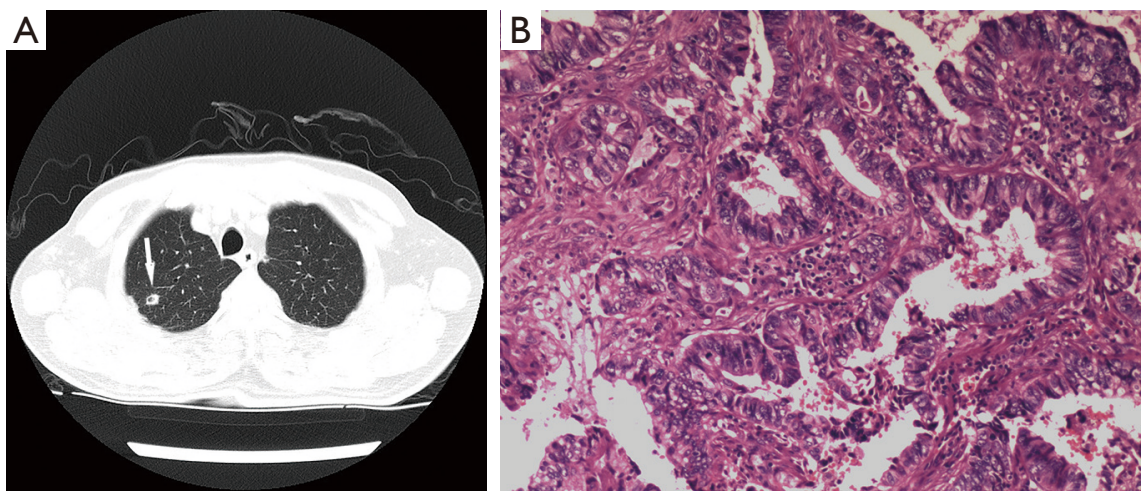

Figure 4 (A) Chest CT scan demonstrating, in horizontal view, an isolated $11 \mathrm{~mm}$ diameter nodule (arrow) in the upper lobe of the right lung. (B) Adenocarcinoma spread to the right lung parenchyma (hematoxylin-eosin staining, magnification $\times 100$ ). Histopathological exam revealed of moderately differentiated tubular adenocarcinoma. Immunohistochemical analysis displayed positive staining for CDX-2 $(+)$, CEA (+), CK20 (+) and Villin (+) with negative staining for CK7 (-) and TTF-1 (-).

radiotherapy was commenced and genetic analysis showed a CODE600 V600E mutation and the tumor was negative for KRAS and NRAS (DT:6000 cGy/30 f, $200 \mathrm{cGy} / \mathrm{f}$ ). After treatment, the patient refused targeted therapy with cetuximab. Post radiotherapy, the patient developed side effects including pharyngoxerosis, dysgeusia (bitter taste), and odynophagia. A chest CT scan showed small nodules suffused in both lungs with the biggest nodule located proximal to the right oblique fissure (Figure 8). Multiple enlarged lymph nodes were present in both the pulmonary hila and the mediastinum with local pleural thickening, all suggesting recurrent disease.

Although chemotherapy is the most appropriate treatment option for metastatic colon cancer, the patient did not consent to treatment principally because of intolerance to previous chemotherapeutic regimens and the fear of further severe allergic reactions. After radiotherapy, the quality of life of the patient significantly declined mainly due to nasosinusitis, tympanitis, hearing loss, pharyngoxerosis, hypogeusia, and restricted mouth opening. During followup after palliative radiotherapy, the patient deteriorated with progressive dyspnoea secondary to the mass-effects of the metastatic deposit in the nasopharynx. The patient eventually died in March 2020 due to dyspnea caused by airway obstruction of the nasopharyngeal mass after 7 months of palliative treatment including pain relief for the end-stage 


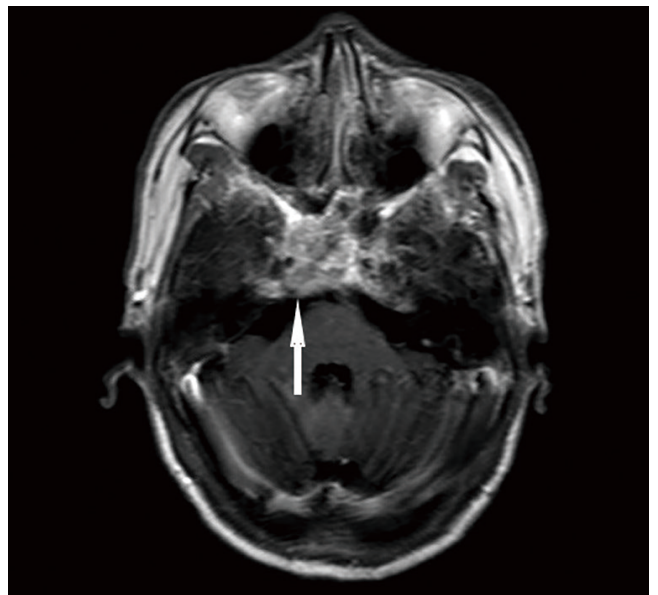

Figure 5 Brain magnetic resonance imaging demonstrating, in the horizontal view, a right bone mass (arrow) in the skull base and clivus invading the parapharyngeal space, cephalic longus muscle, and cavernous sinus (T1W, FLAIR).

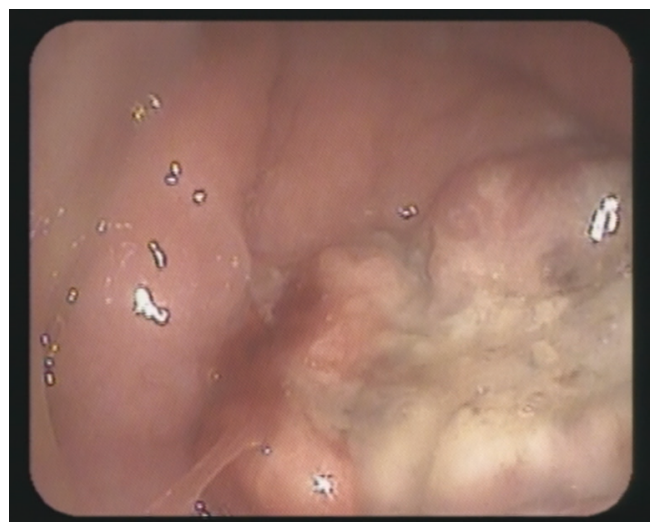

Figure 6 Electronic nasopharyngoscopy illustrating a large number of new cauliflower-like masses in the roof of the posterior wall of the nasopharynx and right pharyngeal recess that had a brittle texture and bled easily during biopsy.

disease.

All procedures performed in studies involving human participants followed the ethical standards of the institutional and/or national research committee(s) and the Helsinki Declaration (revised in 2013). Written informed consent was obtained from the patient. The study was approved by the ethics committee of the first affiliated hospital of Wenzhou Medical University [381-06].

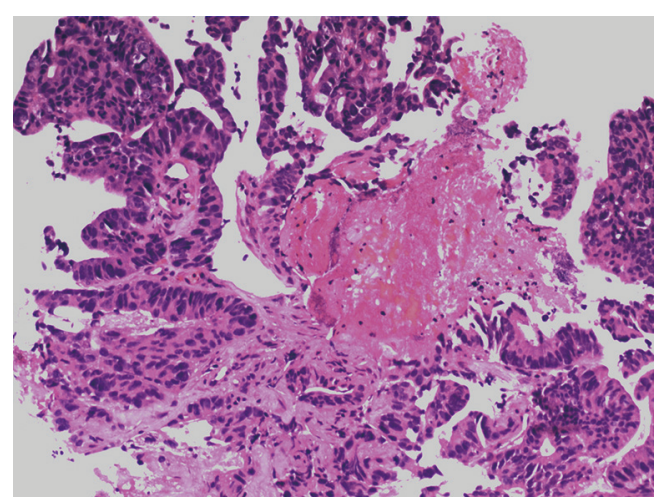

Figure 7 Adenocarcinoma spread to the nasopharyngeal parenchyma (hematoxylin-eosin staining, magnification $\times 100$ ). The histopathological exam revealed adenocarcinoma with extensive necrosis. Immunohistochemical analysis displayed positive staining for CDX-2 (+), TTF-1 (focal +) and Villin (+) with negative staining for CK20 (-) and Napsin A (-).

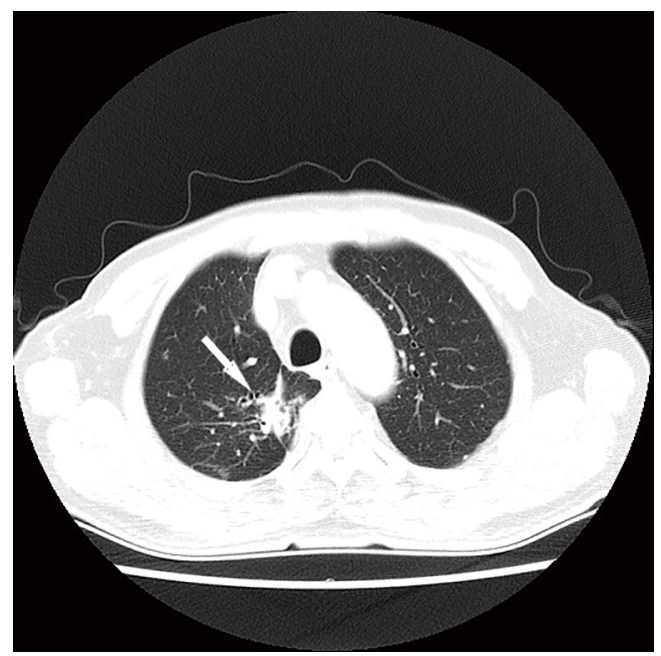

Figure 8 Chest CT scan demonstrating, in the horizontal view, small nodules suffused in both lungs with the largest nodule (arrow) proximal to the right oblique fissure (size $2.5 \times 1.8 \mathrm{~cm}$ ).

\section{Discussion}

Colorectal cancer, commonly known as bowel cancer, comprises malignancies in the colon, rectum, and appendix, and depending on the definition criteria used can also include cancers in the anus. Bowel cancer is classified according to the tumor node metastasis (TNM) staging system into stages I to IV. Cancers that metastasize 
to distal sites (stage-IV) are usually non-curable. The regional lymph nodes, liver, and lungs are the most common sites of metastasis-associated with colorectal cancer. Infrequent sites of metastasis including the spleen, thyroid gland, spermatic cord, and skeletal muscle have also been recorded (1-4).

Although metastases from colorectal cancer to these unusual sites might occur with widespread disease, nasopharyngeal metastasis from colorectal cancer has not yet been reported. To our knowledge, this is the first case of nasopharyngeal metastasis from primary colorectal cancer to be reported in the literature. At present, there are four mechanisms of metastasis linked with colorectal cancer, specifically, lymphatic spread, direct extension, hematogenous spread, and planting spread. Theoretically, based on the analysis of the lymphatic reflux principle and the anatomical location of the nasopharynx, we hypothesize that hematogenous spread of the primary colorectal cancer represents the most likely mechanism of metastasis in this case.

Nasopharyngeal carcinoma often develops at the roof of the nasopharyngeal posterior wall, generally with a cauliflower-like shape and is consistent with our report. Primary cancer is common in nasopharyngeal malignancies making it difficult to determine whether the lesion is metastatic or not. In this context, attention should be paid to the other patient symptoms, signs, and medical history which are essential for accurate diagnosis $(4,5)$.

The main clinical manifestations of nasopharyngeal carcinoma include neck mass (40\%), retracted blood snot $(18.7 \%)$, and ear disorders $(17.0 \%)$. The rate of lymph node metastasis was $82.3 \%$ at the time of diagnosis (6). End-stage patients may present with ocular symptoms due to invasion of the nasopharyngeal into the external ocular muscles and/or the oculomotor nerve (7). Similarly, metastatic nasopharyngeal carcinoma can also cause the previously mentioned symptoms. Histopathological and immunohistochemical examinations enable the identification of the tumor origin in the same tissue. In this case, positive testing for the caudate homologous transcription factor 2 (CDX-2) indicated that the nasopharyngeal neoplasm was a colorectal adenocarcinoma metastasis to the nasopharynx. CDX-2 is a tumor suppressor gene and related studies have implicated the gene in the development and differentiation of normal intestinal epithelial cells during carcinogenesis $(8,9)$.

The established treatment modalities for colorectal carcinoma consist of surgery, chemotherapy, radiotherapy and/or molecular targeted therapies. Radical surgery is the main treatment option, however, postoperative recurrence and metastasis are major causes of poor patient survival. Adjuvant chemotherapy is the primary treatment option following surgery and can reduce the risk of recurrence and metastasis. However, the use of auxiliary therapy in stage IV colorectal cancer patients with primary tumors and metastases who have received local R0 resections remains controversial $(10,11)$.

Based on the findings presented in this case study, chemotherapy did not produce a favorable effect on this elderly patient with nasopharyngeal malignancy. Radiotherapy is the most commonly used and effective treatment modality for head and neck tumors. Except for some severe acute reactions, radiotherapy is well tolerated in the elderly and young patients (12-14).

Over the past 40 years, with the advancement of technology and pharmacotherapy, more effective cancer therapies been developed under integrated clinical management. We conclude that these multiple relapses could be explained by a lack of adjuvant chemotherapy. Our case of atypical colorectal metastasis highlights the significant risk of hematogenous spread that increases with disease progression.

\section{Conclusions}

Nasopharyngeal metastases from colorectal cancer are extremely rare in the clinic. To the best of our knowledge, this is the first case reporting this occurrence that extends our knowledge of the disease and reminds clinicians of the importance of the risk of metastasis during differential diagnosis when head and facial symptoms appear in patients with a history of colorectal cancer. In these cases, nasopharyngoscopy and brain MRI shoulder be performed promptly. The head and facial symptoms cannot be ignored as they may indicate rapid disease progression which correlates with poor prognosis. This case warns doctors to pay more attention to these clinical scenarios. Strict monitoring can give the patients more opportunities for treatments that can provide a better prognosis. Strict monitoring of patients with colorectal cancer after primary treatment can lead to the early diagnosis of metastases and more better opportunities for treatment and increase the probability of better outcomes following treatment.

\section{Acknowledgments}

Funding: This study was funded by the Foundation of 
Wenzhou Municipal Science and Technology Bureau, China (Y20180222).

\section{Footnote}

Reporting Checklist: The authors have completed the CARE reporting checklist. Available at http://dx.doi.org/10.21037/ apm-20-1656

Conflicts of Interest: All authors have completed the ICMJE uniform disclosure form (available at http://dx.doi. org/10.21037/apm-20-1656). The authors have no conflicts of interest to declare.

Ethical Statement: The authors are accountable for all aspects of the work in ensuring that questions related to the accuracy or integrity of any part of the work are appropriately investigated and resolved. All procedures performed in studies involving human participants were in accordance with the ethical standards of the institutional and/or national research committee(s) and with the Helsinki Declaration (as revised in 2013). Written informed consent was obtained from the patient. The study was approved by the ethics committee of the first affiliated hospital of Wenzhou Medical University [381-06].

Open Access Statement: This is an Open Access article distributed in accordance with the Creative Commons Attribution-NonCommercial-NoDerivs 4.0 International License (CC BY-NC-ND 4.0), which permits the noncommercial replication and distribution of the article with the strict proviso that no changes or edits are made and the original work is properly cited (including links to both the formal publication through the relevant DOI and the license). See: https://creativecommons.org/licenses/by-nc-nd/4.0/.

\section{References}

1. Abdou J, Omor Y, Boutayeb S, et al. Isolated splenic metastasis from colon cancer: Case report. World J Gastroenterol 2016;22:4610-4.

2. Hasegawa S, Sakurai Y, Imazu H, et al. Metastasis to the

Cite this article as: Liu YH, Lin BB, Lv SX. Nasopharyngeal metastasis from colorectal cancer: a case report. Ann Palliat Med 2021;10(4):4911-4916. doi: 10.21037/apm-20-1656 forearm skeletal muscle from an adenocarcinoma of the colon: report of a case. Surg Today 2000;30:1118-23.

3. Matsumoto G, Ise H, Inoue H, et al. Metastatic colon carcinoma found within an inguinal hernia sac: report of a case. Surg Today 2000;30:74-7.

4. Phillips JS, Lishman S, Jani P. Colonic carcinoma metastasis to the thyroid: a case of skip metastasis. J Laryngol Otol 2005;119:834-6.

5. Arias-Chamorro B, Galeas-Anaya F, Salinas-Sánchez J, et al. Maxillofacial metastasis of genitourinary origin. A report of 3 cases and review of the literature. Med Oral Patol Oral Cir Bucal 2012;17:e242-5.

6. Liu WW, Guo ZM, Zeng ZY. Clinicopathological features and prognosis of nasopharyngeal adenocarcinoma. Zhonghua Er Bi Yan Hou Tou Jing Wai Ke Za Zhi 2009;44:232-6.

7. Abdel Khalek Abdel Razek A, King A. MRI and CT of nasopharyngeal carcinoma. AJR Am J Roentgenol 2012;198:11-8.

8. Zhang JF, Hu XQ, Ding WF, et al. Expression and its clinical significance of CDX2 and SOX4 in human gastric cancer. J Nantong U (Med Sci) 2019.

9. Li X, Liu S, Yan J, et al. The Characteristics, Prognosis, and Risk Factors of Lymph Node Metastasis in Early Gastric Cancer. Gastroenterol Res Pract 2018;2018:6945743.

10. Gao P, Huang XZ, Song YX, et al. Impact of timing of adjuvant chemotherapy on survival in stage III colon cancer: a population-based study. BMC Cancer 2018;18:234.

11. Liu Q, Luo D, An H, et al. Survival benefit of adjuvant chemotherapy for patients with poorly differentiated stage IIA colon cancer. J Cancer 2019;10:1209-15.

12. Mitsuhashi N, Hayakawa K, Yamakawa M, et al. Cancer in patients aged 90 years or older: radiation therapy. Radiology 1999;211:829-33.

13. Pignon T, Horiot JC, Van den Bogaert W, et al. No age limit for radical radiotherapy in head and neck tumours. Eur J Cancer 1996;32A:2075-81.

14. Zeng Q, Guo X, Li NW, et al. Clinical characteristics and prognosis of aged nasopharyngeal carcinoma patients: a report of 313 cases. Ai Zheng 2008;27:289-94. 